Kompass

Pneumologie

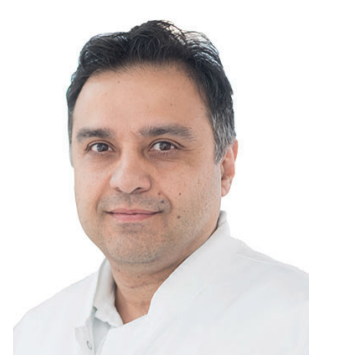

\section{Fotios Drakopanagiotakis}

Medizinische Klinik V, Pneumologie, Klinikum Fulda, Universitätsmedizin Marburg - Campus Fulda, Fulda, Deutschland

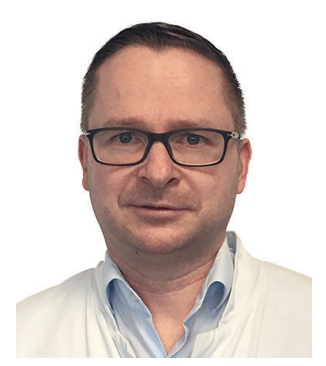

\section{Philipp Markart}

Medizinische Klinik V, Pneumologie, Klinikum Fulda, Universitätsmedizin Marburg - Campus Fulda, Fulda, Deutschland

Medizinische Klinik, Poliklinik II, Universitätsklinikum Gießen und Marburg $\mathrm{GmbH}$, Standort Gießen, Gießen, Deutschland; Mitglied des deutschen Zentrums für Lungenforschung (DZL)

\title{
Seltene Lungenerkrankungen: Symptome deuten und behandeln
}

Als seltene Krankheiten werden in Europa Krankheiten mit einer Prävalenz von weniger als 1:2000 bezeichnet. Seltene Krankheiten sind eine Herausforderung sowohl für die Gesundheitssysteme als auch für die Leistungserbringer. Vor allem sind sie aber eine Herausforderung für die Patienten selbst und ihre Familien, da es häufig zu einer späten und oft zunächst auch falschen Diagnosestellung kommt. Die verspätete Diagnosestellung ist zwangsläufig mit einem verspäteten Behandlungsbeginn assoziiert. Teilweise werden falsche Behandlungen angesetzt, oft stehen auch keine Behandlungsmöglichkeiten zur Verfügung. Die Patienten müssen Spezialisten suchen, die sich sehr oft weit weg von ihrem Wohnort befinden und sie müssen bei Notfällen in Krankenhäusern behandelt werden, die nicht auf die Behandlung ihrer Komplikationen spezialisiert sind [1-4].

Bei Patienten mit Alpha-1-Antitrypsinmangel (AATM) beträgt die Latenz zwischen Auftreten erster klinischer Beschwerden und der Diagnosestellung im Mittel etwa 6 Jahre, bei Patienten mit idiopathischer Lungenfibrose (idiopathic pulmonary fibrosis, IPF) mehr als 1 bis 2,2 Jahre [2, 3].

Warum dauert es so lange, Patienten mit seltenen Lungenerkrankungen zu diagnostizieren? Patienten stellen sich grundsätzlich mit häufigen Symptomen beim Arzt vor: Dyspnoe und Husten. Leider sind diese Symptome unspezifisch: Während der medizinischen Ausbildung lernen wir, die häu- figsten Krankheiten zu erkennen und zu behandeln. Wir erfahren, dass das häufigste auch das Wahrscheinlichste bei unserem Patienten ist. Dieser Ansatz ist für die überwiegende Mehrheit unserer Patienten richtig: Er schützt sie vor unnötiger, potenziell mit Komplikationen einhergehender Diagnostik und das Gesundheitssystem vor ausufernden Kosten. Beispielsweise erscheint eine diffuse Lungenparenchymerkrankung als Ursache des Hustens erst am Ende des empfohlenen diagnostischen Algorithmus und erst nachdem die diagnostischen und therapeutischen Maßnahmen zu den häufigen Ursachen ausgeschöpft sind (Asthma bronchiale, gastroösophagealer Reflux und Postnasal-Drip-Syndrom). Ferner wird in den Hustenleitlinien empfohlen - bei fehlenden bedrohlichen klinischen Zeichen die Diagnostik des Hustens bei anhaltenden Symptomen erst nach einer Dauer von 3 Monaten zu beginnen [5].

Darüber hinaus ist es praktisch unmöglich, dass jeder Arzt die etwa 8000 seltenen Krankheiten kennt, die es in Deutschland gibt und die im Netzwerk der seltenen Krankheiten Orphanet aufgezeichnet werden. Zudem sind unsere Kenntnisse bei seltenen Lungenerkrankungen oft falsch: In einer Studie unter Allgemeinmedizinern in Europa gaben $41 \%$ von ihnen an, dass sie Patienten mit seltenen Lungenerkrankungen behandeln. Die Verwendung von Fragebögen ergab jedoch einen Mangel an allgemeinem Wissen über seltene Lun- information@karger.com

() 2020 S. Karger GmbH, Freiburg

www.karger.com/kkp

Karger ${ }^{\prime}=$
Dr. Fotios Drakopanagiotakis

Klinikum Fulda

Pacelliallee 4, 36043 Fulda, Deutschland

fotios.drakopanagiotakis@klinikum-fulda.de 
generkrankungen: Zum Beispiel glaubten 80\%, dass ein erhöhter, statt reduzierter A1Antitrypsin-Spiegel das Kennzeichen von AATM ist. 79\% der Befragten antworteten, dass bilaterale Pleuraergüsse das Hauptsymptom bei Patienten mit Mukoviszidose sind [6].

Auch unter den Pneumologen gibt es große Unterschiede in ihrer Erfahrung und ihrem Wissen bei der Diagnose seltener Krankheiten: In einer internationalen Studie unter Einschluss von etwa 400 Pneumologen gab es erhebliche Unterschiede bezüglich der Diagnose bei interstitiellen Lungenerkrankungen (interstitial lung diseases, ILDs) zwischen erfahrenen und weniger erfahrenen Pneumologen [7].

Ein weiterer Faktor, der die Behandlung von Patienten mit seltenen Lungenerkrankungen erschwert, ist das Auftreten neuer Symptome unter Therapie. Unsere Erfahrung bezüglich Nebenwirkungen und Wechselwirkungen dieser neuen Medikamente ist begrenzt: Es ist z.B. nicht einfach zu unterscheiden zwischen einer medikamentösen Nebenwirkung und einem Progress der Grunderkrankung bei einem Patienten mit interstitieller Lungenerkrankung auf dem Boden einer rheumatologisch-immunologischen Systemerkrankung unter Biologika, der sich mit Dyspnoeverschlechterung und alveolären Infiltraten präsentiert [4].

Können wir unsere Effektivität bei der Diagnosestellung seltener Lungenerkrankungen auf der Grundlage von Symptomen und klinischer Untersuchung verbessern?
Es gibt schon solche Beispiele: Die Assoziation einer Sklerosiphonie mit idiopathischen diffusen Lungenerkrankungen hat zu vermehrten Überweisungen von Hausärzten zu Pneumologen geführt [8].

\section{Während Netzwerke von grundlegender Bedeutung für die Wissenschaft sind und als Referenz für Diagnose- und Management-Leitlinien dienen, sind Hausärzte die ersten Kontaktpersonen im Gesundheits- wesen, die Patienten mit seltenen \\ Lungenerkrankungen erkennen und im Gesundheitssystem begleiten müssen.}

Eine wichtige Initiative der Europäischen Kommission seltene Krankheiten betreffend ist die Gründung europäischer Referenznetzwerke (European Reference Network, ERN). Zudem schließen zahlreiche Register und Biobanken für seltene Lungenerkrankungen Patienten ein, mit dem Ziel, die Symptome und den klinischen Verlauf der Krankheiten besser erkennen zu können. Während Netzwerke von grundlegender Bedeutung für die Wissenschaft sind und als Referenz für Diagnose- und Management-Leitlinien dienen, sind Hausärzte (sowohl für pädiatrische als auch für erwachsene Patienten) die ersten Kontaktpersonen im Gesundheitswesen, die Patienten mit seltenen Lungenerkrankungen erkennen und im Gesundheitssystem begleiten müssen. Die Hausärzte sind ebenso wichtige Ansprechpartner zwischen den Besuchen beim Spezialisten. In diesem Zusammenhang gibt es Initiativen der European Respiratory Society (ERS) und der European Lung Foundation (ELF) für die Förderung von Bildungsaktivitäten in ganz Europa, um das Bewusstsein für seltene Lungenkrankheiten, auch bei Hausärzten, zu erhöhen [6].

Eine entscheidende Rolle der Patientenverbände bei seltenen Erkrankungen wurde in den letzten Jahren zunehmend erkannt. Eine starke Partnerschaft mit medizinischen Gesellschaften wie der ERS ist maßgeblich für die Etablierung erfolgreicher politischer Maßnahmen und für die Verbesserung der Kommunikation zwischen Angehörigen der Gesundheitsberufe, Patienten mit seltenen Krankheiten und ihren Angehörigen $[9,10]$.

Das nun vorliegende Heft von «Kompass Pneumologie» soll Ihnen mit seinen Fokusbeiträgen einige Aspekte der Symptomeninterpretation bei seltenen Lungenerkrankungen nahebringen.

Wir wünschen Ihnen eine anregende Lektüre

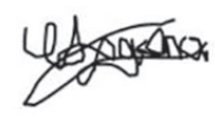

Dr. Fotios Drakopanagiotakis

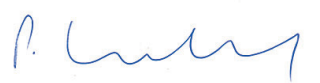

Prof. Dr. Philipp Markart

\section{Literatur}

1 Pritchard D, Adegunsoye A, Lafond E, et al.: Diagnostic test interpretation and referral delay in patients with interstitial lung disease. Respir Res. 201912;20(1):253.

2 Moor CC, Wijsenbeek MS, Balestro E, et al.: Gaps in care of patients living with pulmonary fibrosis: a joint patient and expert statement on the results of a Europe-wide survey. ERJ Open Res. 2019;5(4):00124-2019.

$\checkmark 3$ Wencker M, Teschler H, Vogelmeier C, et al.: Rare diseases in pulmonary medicine and its challenges. Pneumologie. 2012;66(7):437-441.

$\checkmark 4$ Bendstrup E, Møller J, Kronborg-White S, et al.: Interstitial Lung Disease in Rheumatoid
Arthritis Remains a Challenge for Clinicians. J Clin Med. 2019;8(12):pii:E2038.

5 Kardos P, Dinh Q-T, Fuchs K-H, et al.: Leitlinie der Deutschen Gesellschaft für Pneumologie und Beatmungsmedizin zur Diagnostik und Therapie von erwachsenen Patienten mit Husten. Pneumologie. 2019;73:143-180.

-6 Balbi B, Vallese D, Chavannes N, et al.: General practicioners and rare lung diseases: a task fo the development of rare lung diseases educational material. Breathe (Sheff). 2016; 12(4):341-348.

7 Walsh SLF, Maher TM, Kolb M et al: Diagnostic accuracy of a clinical diagnosis of idiopathic pulmonary fibrosis: an international case-cohort study. Eur Respir J. 2017;50(2):pii:1700936

$>8$ Purokivi M, Hodgson U, Myllärnemi M: Are physicians in primary health care able to recognize pulmonary fibrosis? Eur Clin Respir J. 2017;4(1):1290339.

-9 McCarthy C, Lara Gallego B, Trapnell BC, et al.: Epidemiology of Rare Lung Diseases: The Challenges and Opportunities to Improve Research and Knowledge. Adv Exp Med Biol. 2017;1031:419-442.

10 Harari S, Humbert M: Rare pulmonary diseases: a common fight. Eur Respir Rev. 2017; 26(145):pii:170059. 